Bulletin of the Section of Logic

Volume 45:3/4 (2016), pp. 213-238

http://dx.doi.org/10.18778/0138-0680.45.3.4.06

Bogdan Staruch

\title{
IRREDUNDANT DECOMPOSITION OF ALGEBRAS INTO ONE-DIMENSIONAL FACTORS
}

\begin{abstract}
We introduce a notion of dimension of an algebraic lattice and, treating such a lattice as the congruence lattice of an algebra, we introduce the dimension of an algebra, too. We define a star-product as a special kind of subdirect product. We obtain the star-decomposition of algebras into one-dimensional factors, which generalizes the known decomposition theorems e.g. for Abelian groups, linear spaces, Boolean algebras.

Keywords: universal algebra, algebraic lattice, congruence lattice, uniform lattice, dimension of algebra, one-dimensional algebra, subdirect product, star-product, decomposition of algebra
\end{abstract}

\section{Introduction}

In this paper we study properties of algebraic lattices in order to obtain subdirect decomposition of algebras into a minimal number of irreducible factors. The famous Grätzer-Schmidt theorem (see [6]) states that every algebraic lattice is the congruence lattice of an algebra, so we study algebraic lattices having in mind congruence lattices. Our main aim was to generalize the known (in classical algebra) decomposition theorems to universal algebra. For example, there are theorems on decomposition: of vector spaces into one dimensional vector spaces, of finitely generated Abelian groups into irreducible cyclic groups, or finitely generated Boolean algebras into two-element Boolean algebras. 
In the context of decomposition of algebras, it is known the Birkhoff's Theorem (see [1]) which states that every algebra $\mathbf{A}$ is isomorphic to a subdirect product of subdirectly irreducible algebras, but this theorem is redundant and too general. To get irredundant decompositions we introduce the notion of dimension of an algebraic lattice. We don't know if every algebraic lattice has the dimension, but we show that every balanced algebraic lattice has the dimension (see Theorem 3.10), and there are also non-balanced lattices with dimension (see Example 2.21). We define the dimension of an algebra as the dimension of its congruence lattice. This notion of dimension generalizes the so called Goldie dimension, which is well defined in locally uniform lattices which are at least balanced. We show in this paper that in balanced locally uniform lattices Goldie dimension is equal to our dimension. Goldie (see [4]) used the notion of uniform modules to construct uniform dimension (Goldie dimension) of a module. Goldie dimension generalizes some, but not all, aspects of the notion of the dimension of a vector space. Goldie dimension in modular lattices were studied by Grzeszczuk, Okniński and Puczyłowski in [8], [9], [7], [14], and in balanced lattices - by Zolotarev and Krempa in [18], [19], [11], [12]. We use terminology from these works such as uniform element, essential element, independent set, balanced lattice.

We also observed that in some known decomposition theorems, for example in finitely generated Abelian groups, there are irreducible factors, namely the group of integers $Z$, that are not subdirectly irreducible. Such algebras are finitely subdirectly irreducible. This kind of algebras is known in the context of decomposition of algebras, for example see the paper of Katriñák and El-Assar [10]. It is easy to see that every subdirectly irreducible algebra is also finitely subdirectly irreducible. Finitely subdirectly irreducible algebras are exactly one-dimensional algebras.

We introduced in this paper some new terminology and techniques. The first tool is the omitting relation necessary in almost all proofs and in definitions of $\circledast$-sets and $\circledast$-products, which define the decomposition mentioned at the beginning. $\circledast$-sets and maximal independent sets are used in the definition of a basis of an algebraic lattice and the dimension of an algebra. We also introduced the notion of an anti-uniform element in a lattice. In algebras with dimension we obtain a $\circledast$-decomposition into one-dimensional algebras with number of factors equal to the dimension of this algebra and, in some cases, a one algebra related to anti-uniform part of the congruence lattice needs to be included in this $\circledast$-decomposition. 
The most interesting results are in the case of modular lattices, where we obtained a full $\circledast$-decomposition (see Theorem 4.12) of algebras. This theorem applies to many algebraic structures like modules, groups and rings (noncommutative included) as their congruence lattices are modular. In the case of congruence distributive algebras the $\circledast$-decompositions (see Theorem 4.16) are unique. This theorem applies to algebraic structures such as lattices, Boolean algebras and Heyting algebras.

\subsection{Preliminaries}

An algebra $\mathbf{A}$ of type $F$ is an ordered pair $(A, F)$, where $A$ is a nonempty set and $F$ is a family of finitary operations on $A$.

A binary relation $\theta$ on $A$ is called a congruence on an algebra $\mathbf{A}$ of type $F$ if it is an equivalence relation on $A$ satisfying the compatibility property i.e. for each $n$-ary operation $f \in F$ and elements $a_{i}, b_{i} \in A$ if $\left(a_{i}, b_{i}\right) \in \theta$ holds for $i=1, \ldots, n$ then $\left(f\left(a_{1}, \ldots, a_{n}\right), f\left(b_{1}, \ldots, b_{n}\right)\right) \in \theta$. The set of all congruences on an algebra $\mathbf{A}$ is denoted by $\operatorname{Con} \mathbf{A}$. It is known that $\operatorname{Con} \mathbf{A}$ ordered by inclusion is an algebraic lattice which is called the congruence lattice of $\mathbf{A}$. The least element 0 is the identity relation and the greatest element 1 is $A \times A$. According to terminology like 'congruence modular algebra' and 'congruence distributive algebra', we will say that algebra is a congruence 'Property' algebra if its congruence lattice has the property 'Property'.

An algebra $\mathbf{A}$ is a subdirect product of a family $\left(\mathbf{A}_{i}\right)_{i \in I}$ of algebras if $\mathbf{A}$ is a subalgebra of the product $\prod\left(\mathbf{A}_{i}\right)_{i \in I}$ and the projection maps $\pi_{i}: \mathbf{A} \rightarrow \mathbf{A}_{i}$ are epimorphisms for each $i \in I$.

We use two important properties of congruences. The first one states that if $\theta=\bigcap\left\{\theta_{i}\right\}_{i \in I}$, then $\mathbf{A} / \theta$ is a subdirect product of the algebras $\left(\mathbf{A} / \theta_{i}\right)_{i \in I}$. Hence if $0=\bigcap\left\{\theta_{i}\right\}_{i \in I}$, then $\mathbf{A}$ is a subdirect product of the quotient algebras $\left(\mathbf{A} / \theta_{i}\right)_{i \in I}$. The second one is The Correspondence Theorem which states that for any algebra $\mathbf{A}$ and $\theta \in \operatorname{Con} \mathbf{A}$ the interval $[\theta, 1]$ is isomorphic to $\operatorname{Con} \mathbf{A} / \theta$.

An algebra $\mathbf{A}$ is finitely subdirectly irreducible iff 0 is a meet irreducible element in $\operatorname{Con} \mathbf{A}$. If, additionaly, 0 is a completely meet irreducible element in $\operatorname{Con} \mathbf{A}$, then $\mathbf{A}$ is called subdirectly irreducible. Every algebra with an atomic congruence lattice with one atom is subdirectly irreducible.

For facts not recalled here see [3], [2], [5], [13]. 


\section{General case - algebraic lattices}

We assume throughout the paper, that $L$ is an algebraic lattice with the least element denoted by 0 and the greatest element denoted by 1 , and where $0 \neq 1$. This yields that if such an $L$ is a congruence lattice of an algebra $\mathbf{A}$, then $\mathbf{A}$ is nontrivial, i.e. it has at least two elements.

If $L$ is algebraic then it is complete by definition. In complete lattices we have $\bigvee \emptyset=0, \bigwedge \emptyset=1, \bigvee L=1, \bigwedge L=0$

We use the fact that every algebraic lattice is upper continuous (see [15]), i.e. for every up-directed subset $D \subseteq L$ and $a \in L, \quad a \wedge \vec{V} D=\vec{\bigvee}_{d \in D}(a \wedge d)$, where $\vec{\vee} D$ denotes the join of an up-directed set $D$ in $L$.

\subsection{Independent sets, omitting relation and star-products}

The idea of introducing independent sets has its origin in independent sets of vectors in vector spaces. There are several definitions of independent sets in lattices (see e.g. [12], [18], [7], [5]). We use the following:

Definition 2.1. A subset $X \subseteq L \backslash\{0\}$ is independent if $|X|=1$, or $|X|>1$ and for every $x \in X$ it holds that $x \wedge \bigvee(X \backslash\{x\})=0$.

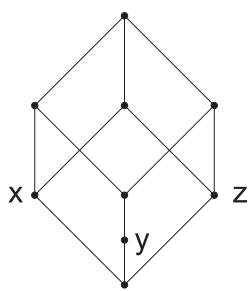

Lattice 1

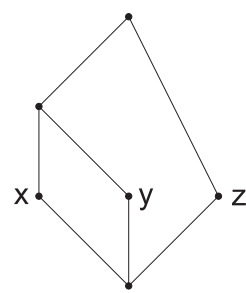

Lattice 2

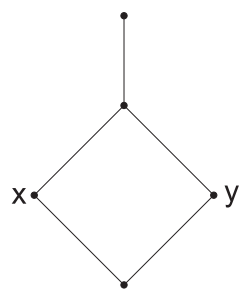

Lattice 3

Fig. 1.

The definition given in Grätzer's book [5] is that an $X \subseteq L \backslash\{0\}$ is independent iff $\bigvee X_{1} \wedge \bigvee X_{2}=\bigvee\left(X_{1} \cap X_{2}\right)$ for any two finite subsets $X_{1}, X_{2} \subseteq X$. Notice that if $X$ is independent in the sense of Grätzer's definition in an algebraic lattice then it is independent in the sense of 
Definition 2.1 in this lattice. However, the opposite doesn't hold. For example, the set $X=\{x, y, z\}$ in Lattice 1 (see Fig. 1.) is independent, but it is not independent in the sense of Grätzer's definition, because $\bigvee\{x, y\} \wedge$ $\bigvee\{y, z\}>\bigvee\{y\}=y$.

Proposition 2.2. Let $X \subseteq L$ and $|X|>1$. Then $X$ is an independent set iff every finite nonempty subset $Y \subseteq X$ is independent.

Proof. $\Rightarrow$ is trivial. To show $\Leftarrow$ notice that for any $x \in X, \bigvee(X \backslash\{x\})=$ $\vec{\bigvee} D$, where $D$ is an up-directed set of all the finite joins of elements from $X \backslash\{x\}$, and $x \wedge d=0$ for every $d \in D$. Hence $x \wedge \bigvee(X \backslash\{x\})=$ $\vec{\bigvee}_{d \in D}(x \wedge d)=0$ for any $x \in X$.

Now, we introduce a kind of complementation, namely, the relation of omitting of subsets, which plays a crucial role in this paper. We say that an element $y \in L$ omits $x \in L$ iff $y \wedge x=0$. An element $y \in L$ omits a nonempty subset $X \subseteq L$ iff $y$ omits every element $x \in X$. If $X=\emptyset$ then every element $y \in L$ omits $X$.

Using Zorn's Lemma it can be shown that for any subset $X \subseteq L$ (the assumption that $L$ is an algebraic lattice is important) there exists a maximal element omitting $X$. The set of all maximal elements omitting $X$ is denoted by $\mathfrak{M}_{X}$ and elements of this set are denoted by $M_{X}$. If $X=\{x\}$ then the set of all maximal elements omitting $x$ is denoted by $\mathfrak{M}_{x}$ and $M_{x}$ denotes any element of $\mathfrak{M}_{x}$.

We consider also maximal elements in $L$ omitting a given element $M_{X}$ using notation $M_{X}^{\circledast}$ for any element of the set $\mathfrak{M}_{M_{X}}$. Exceptionally, if $X=$ $\{x\}$ then for a given $M_{x}, M_{x}^{\circledast}$ denotes any element of the set $\mathfrak{M}_{x}^{\circledast}\left(M_{x}\right)=$ $\left\{z \in \mathfrak{M}_{M_{X}}: x \leq z\right\}$. Consequently, for given $M_{X}$ and $M_{X}^{\circledast} \in \mathfrak{M}_{M_{X}}$, the symbol $M_{X}^{\circledast \circledast}$ denotes any element of the set $\mathfrak{M}_{M_{X}}^{\circledast}\left(M_{X}^{\circledast}\right)$. Similarly, for given $M_{X}, M_{X}^{\circledast} \in \mathfrak{M}_{M_{X}}$ and $M_{X}^{\circledast \circledast} \in \mathfrak{M}_{M_{X}}^{\circledast}\left(M_{X}^{\circledast}\right)$, the symbol $M_{X}^{\circledast \circledast \circledast}$ denotes any element of $\mathfrak{M}_{M_{X}^{\circledast}}^{\circledast}\left(M_{X}^{\circledast \circledast}\right)$.

Look at the following intuitive example, where $L$ is a lattice of subspaces of a linear space $V$. Let $\emptyset \neq X \subseteq L$ and let $s=\bigvee X$. Then $M_{s}^{\circledast}$ is the subspace generated by $X$ and $M_{s}$ is a completion subspace such that the direct sum of $M_{s}^{\circledast}$ and $M_{s}$ is $V$.

Proposition 2.3. 1. If $x, y \in L$ and $x \leq y$, then for any $M_{y}$ there is an $M_{x}$ such that $M_{y} \leq M_{x}$.

2. Let $x, y \in L$ and $x \leq y$. If $M_{x}$ omits $y$ then $M_{x}=M_{y}$ for some $M_{y}$. 
3. For any $M_{x}, M_{x}=M_{x}^{\circledast \circledast}$ independently of the choice of $M_{x}^{\circledast}$. Moreover, for any $X \subseteq L$ and $M_{X}^{\circledast}, M_{X}^{\circledast}=M_{X}^{\circledast \circledast \circledast}$ independently of the choice of $M_{X}^{\circledast \circledast}$.

4. Let $\emptyset \neq X \subseteq L$ and $s=\bigvee X$. Then for every $M_{s}$ there exists a choice of $\left\{M_{x}\right\}_{x \in X}$ such that $M_{x} \geq M_{s}$ and then $M_{s} \leq \bigwedge\left\{M_{x}\right\}_{x \in X}$.

5. Let $\emptyset \neq X \subseteq L$. Then for any $M_{X}$ there exists a choice of $\left\{M_{x}\right\}_{x \in X}$ such that $M_{X}=\bigwedge\left\{M_{x}\right\}_{x \in X}$.

Proof. (i) and (ii) are obvious.

(iii) By definition $x \leq M_{x}^{\circledast}, M_{x} \leq M_{x}^{\circledast \circledast}$ and $M_{x}^{\circledast \circledast}$ omits $x$. Thus $M_{x}=$ $M_{x}^{\circledast \circledast}$ by maximality of $M_{x}$. To show the second part substitute $M_{X}$ in the place of $x$ in the first part.

(iv) Notice that for any $x \in X, x \leq s$ and use (i).

(v) Take $M_{X}$ and any $x \in X$. Then $M_{X}$ omits $x$, so there is $M_{x}$ such that $M_{X} \leq M_{x}$ by maximality of $M_{x}$. Hence $M_{X} \leq \bigwedge\left\{M_{x}\right\}_{x \in X}$ and $\bigwedge\left\{M_{x}\right\}_{x \in X}$ omits $X$. Maximality of $M_{X}$ yields the equality.

Corollary 2.4. For any algebra $\mathbf{A}$, if $\emptyset \neq X \subseteq$ Con $\mathbf{A}$ then for any $M_{X}$ there exists a choice of $\left\{M_{x}\right\}_{x \in X}$ such that $\mathbf{A} / M_{X}$ is isomorphic to a subdirect product of algebras $\left(\mathbf{A} / M_{x}\right)_{x \in X}$.

The above decomposition has too many factors. We aim to obtain more specific irredundant decomposition of algebras, so we need to introduce some more definitions and new terminology.

An element $e \in L$ is called essential in $L$ if $x \wedge e \neq 0$ for every $x \in L \backslash\{0\}$. This notion is known in module theory, where Goldie bases were introduced in submodule lattices. The greatest element 1 is essential in every lattice with 0 and 1 . If $x$ is essential in $L$ then $M_{x}=0$. We say that an element $e \in L$ is essential in an element $a \in L$ iff $e \leq a$ and $e$ is essential in the interval $[0, a] \subseteq L$.

Definition 2.5. Let $X, Y \subseteq L$ and $X=\left\{x_{i}\right\}_{i \in I}, Y=\left\{y_{i}\right\}_{i \in I}$. We say that the pair $(X, Y)$ is a star pair (a $\circledast$-pair) in $L$ iff

1. $X$ is an independent set in $L$ such that $\bigvee X$ is essential in $L$,

2. $\bigwedge Y=0$,

3. $y_{i}$ is a maximal element omitting $x_{i}$ such that $\bigvee\left(X \backslash\left\{x_{i}\right\}\right) \leq y_{i}$ is essential in $y_{i}$, for every $i \in I$. 
Notice that if $|I|=1$, then the only $\circledast$-pair is $(\{1\},\{0\})$. If $|I|>1$ and $(X, Y)$ is a $\circledast$-pair in $L$ then there exists a choice of elements $\left\{M_{i}^{X}\right\}_{i \in I}$ such that for every $i \in I, M_{i}^{X}=y_{i}$. In Lattice 3 (see Fig. 1.) $x \vee y \neq 1$ is essential and $(\{x, y\},\{x, y\})$ is a $\circledast$-pair.

ExAmple 2.6. 1. Let $\mathbf{A}=\mathbf{A}_{1} \times \mathbf{A}_{2}$ be a direct product of nontrivial algebras $\mathbf{A}_{1}, \mathbf{A}_{2}$. Let $X=Y=\left\{k e r \pi_{1}, k e r \pi_{2}\right\}$. Then $(X, Y)$ is a $\circledast$-pair in $\operatorname{Con} \mathbf{A}$.

2. Let $\mathbf{A}=\Pi\left(\mathbf{A}_{i}\right)_{i \in I}$ be a direct product of a finite family of nontrivial algebras $\left(\mathbf{A}_{i}\right)_{i \in I}, I=\{1,2, \ldots, n\}$. Then the pair $(X, Y)$, where $Y=\left\{k e r \pi_{i}\right\}_{i \in I}$ and $X=\left\{\bigwedge\left\{k e r \pi_{j}\right\}_{j \in I \backslash\{i\}}\right\}_{i \in I}$ is a $\circledast$-pair in ConA.

Before we formulate the next example, notice that any vector space $\mathbf{V}$ over a field $K$ can be represented as an algebra determined on $V$ with the operation of adding vectors and a set of unary operations $\left\{f_{k}\right\}_{k \in K}$ such that $f_{k}(v)=k v$. This algebra has its congruence lattice isomorphic to its subalgebra lattice and, to the subspace lattice of $\mathbf{V}$, as well. Many algebraic structures with external operations can be represented as algebras in this way.

EXAmPle 2.7. Let $\mathbf{V}=\bigoplus\left(\mathbf{V}_{i}\right)_{i \in I}$ be a direct sum of nontrivial vector subspaces. Let $X=\left(\mathbf{X}_{i}\right)_{i \in I}$, where for every $i \in I, \quad \mathbf{X}_{i}$ is a subspace of $\mathbf{V}$ such that $X_{i}=\left\{v \in V: v_{j}=0\right.$ for every $\left.j \neq i\right\}$. Let $Y=\left\{\mathbf{Y}_{i}\right\}_{i \in I}$, where for every $i \in I, \quad \mathbf{Y}_{i}$ is a subspace of $\mathbf{V}$ such that $Y_{i}=\left\{v \in V: v_{i}=0\right\}$. Then $(X, Y)$ is a $\circledast$-pair in the lattice of subspaces of $\mathbf{V}$.

We will use notation $\bar{y}=\bigwedge(Y \backslash\{y\})$ and $\bar{Y}=\{\bar{y}: y \in Y\}$.

Definition 2.8. We say that $Y=\left\{y_{i}\right\}_{i \in I}$ is a star-set (a $\circledast$-set) in $L$ iff $(\bar{Y}, Y)$ is a $\circledast$-pair in $L$.

Notice that in all cases in Examples 2.6 and 2.7, $X=\bar{Y}$ and $Y$ is a $\circledast$-set in the appropriate lattice.

It is worth mentioning that every $\circledast$-set $Y$ is irredundant, that is, $\bigwedge Y=$ 0 and $\bigwedge(Y \backslash\{y\})=\bar{y} \neq 0$ for any $y \in Y$. Moreover, if $|Y|>1$ then $y \neq 0$ and $y$ is not essential in $L$ for every $y \in Y$.

Definition 2.9. An algebra $\mathbf{A}$ is called a star-product (a $\circledast$-product) of algebras $\left(\mathbf{A}_{i}\right)_{i \in I}$ if $\mathbf{A}$ is a subdirect product of this family such that the set $Y=\left\{k e r \pi_{i}\right\}_{i \in I}$ of congruences on $\mathbf{A}$ is a $\circledast$-set. 
If $\mathbf{A}$ is a $\circledast$-product of algebras $\left(\mathbf{A}_{i}\right)_{i \in I}$ we use notation $\circledast\left(\mathbf{A}_{i}\right)_{i \in I}$. Corollary 2.10. If $Y=\left\{\theta_{i}\right\}_{i \in I}$ is a $\circledast$-set in Con $\mathbf{A}$ then $\mathbf{A} \simeq \circledast\left(\mathbf{A}_{i}\right)_{i \in I}$.

\subsection{Uniform and anti-uniform elements}

We recall here the very important definition of a uniform element in a lattice, known from papers of Grzeszczuk and Puczyłowski [8], [9].

We say that an element $u \in L \backslash\{0\}$ is uniform in $L$ if $x \wedge y \neq 0$ for any $x, y \in(0, u]$. The lattice $L$ is uniform if 1 is uniform. The set of all uniform elements in $L$ will be denoted by $U$. We omit the subscript $L$ because the lattice will be known from the context. The set of all atoms will be denoted by $A t$. Every atom is uniform, so $A t \subseteq U$.

Every chain lattice is uniform, the congruence lattice of any finitely subdirectly irreducible algebra is uniform and the lattice of all subgroups of the group of integers $Z$ is uniform. The lattice of all subgroups of the group $Z \times Z$ is not uniform. Generally, the lattice of all subgroups of the power $Z^{n}, n \geq 2$ is not uniform but there are infinitely many uniform elements in $Z^{n}$.

FACT 2.11. Let $u \in U$ in $L$. Then:

1. $u$ is uniform in every interval $[0, v]$ such that $v \geq u$,

2. if $0<x \leq u$ then $x$ is uniform in $L$,

3. for any $x \in L$ if $x \wedge u \neq 0$ then $x \wedge u$ is uniform in $L$.

Proposition 2.12. Let $u, v \in U \neq \emptyset$ and $u \leq v$. Then for every $x \in L$, $x \wedge u=0$ iff $x \wedge v=0$, and thus $\mathfrak{M}_{u}=\mathfrak{M}_{v}$.

Proof. Obviously, if $x \wedge v=0$ then $x \wedge u=0$. Assume that $x \wedge u=0$. If $x \wedge v \neq 0$ then $0 \neq u \wedge(x \wedge v)=x \wedge(u \wedge v)=x \wedge u$. By Proposition 2.3 for any $M_{v}$ there exists an $M_{u}$ such that $M_{v} \leq M_{u}$. If $M_{v}<M_{u}$, then $M_{u}$ doesn't omit $v$, so $0 \neq M_{u} \wedge v \leq v$. Hence $0 \neq\left(M_{u} \wedge v\right) \wedge u=M_{u} \wedge u=0$. This contradiction yields $M_{v}=M_{u}$. Now, let us take any $M_{u}$. Then $0=M_{u} \wedge u=\left(M_{u} \wedge v\right) \wedge u$, and since $u$ is uniform, $M_{u} \wedge v=0$. So, $M_{u} \leq M_{v}$. If $M_{u}<M_{v}$, then $M_{v}$ doesn't omit $u$, which yields $M_{u}=M_{v}$.

FACT 2.13. 1. A congruence $\theta \neq 1$ in $\operatorname{Con} \mathbf{A}$ is meet irreducible iff the interval $[\theta, 1]$ is a uniform lattice iff the quotient algebra $\mathbf{A} / \theta$ is finitely subdirectly irreducible. 
2. A congruence $\theta \neq 1$ in $\operatorname{Con} \mathbf{A}$ is completely meet irreducible iff the interval $[\theta, 1]$ is an atomic lattice with exactly one atom iff the quotient algebra $\mathbf{A} / \theta$ is subdirectly irreducible.

Theorem 2.14. Let $u \in U$ and $a \in A$. Then for any $M_{u}$ and $M_{a}$ the interval $\left[M_{u}, 1\right]$ is uniform, while the interval $\left[M_{a}, 1\right]$ is atomic with exactly one atom.

Proof. Let $x, y \in\left(M_{u}, 1\right]$. Then $M_{u} \leq x \wedge y$. Thus, by maximality of $M_{u}, x \wedge u \neq 0 \neq y \wedge u$. As $u$ is uniform, $(x \wedge u) \wedge(y \wedge u)=(x \wedge y) \wedge u \neq 0$. Hence $M_{u}<x \wedge y$, so $\left[M_{u}, 1\right]$ is uniform. If $a$ is an atom, then $a \leq x$ for every $x>M_{a}$, so $M_{a} \leq \bigwedge\left\{x \in L: x>M_{a}\right\} \geq a$. Thus $\bigwedge\left\{x \in L: x>M_{a}\right\}$ doesn't omit $a$ and this yields $M_{a}<\bigwedge\left\{x \in L: x>M_{a}\right\}$.

Corollary 2.15. Let $u \in U$ and $a \in A t$ in Con $\mathbf{A}$ for some algebra $\mathbf{A}$. Then for any $M_{u}, M_{a}$ in $\operatorname{Con} \mathbf{A}, \mathbf{A} / M_{u}$ is finitely subdirectly irreducible and $\mathbf{A} / M_{a}$ is subdirectly irreducible.

Let us introduce here a new notion of an anti-uniform element in $L$. We say that an element $t \in L \backslash\{0\}$ is anti-uniform in $L$ if there is no uniform element in the interval $[0, t]$. Hence every anti-uniform element omits $U$. In other words, $t$ is anti-uniform if for every $0<s \leq t$ there exist nonzero elements $x, y \leq s$ such that $x \wedge y=0$. A lattice $L$ with 0 and 1 is anti-uniform if 1 is anti-uniform. The set of all anti-uniform elements in $L$ is denoted by $T$. It is easy to see that every nonzero $M_{U}$ is a maximal antiuniform element in $L$. The Lindenbaum-Tarski algebra with an infinite set of variables is an anti-uniform lattice.

FACT 2.16. Let $t$ be anti-uniform in a lattice $L$. Then:

1. $t$ is anti-uniform in every interval $[0, v]$ such that $v \geq t$,

2. if $0<x \leq t$ then $x$ is anti-uniform in $L$,

3. for any $x \in L$ if $x \wedge t \neq 0$ then $x \wedge t$ is anti-uniform in $L$.

FACT 2.17. $M_{U} \wedge M_{T}=0$ for any $M_{U}$ and $M_{T}$.

We say that a lattice $L$ is locally uniform if for every $x \in L \backslash\{0\}$ there exists a uniform element $u$ such that $u \leq x$. Atomic lattices and, in consequence, finite lattices, the lattice of all subgroups of the power $Z^{n}$, the lattice of subspaces of the linear space $R^{n}$ are locally uniform. 
Notice that if $L$ is locally uniform then $M_{U}=0, M_{U}^{\circledast}=M_{T}=1$. If $L$ is anti-uniform then $M_{U}=1, M_{U}^{\circledast}=M_{T}=0$. Moreover, if $L$ is atomic then $M_{A}=M_{U}=0, M_{U}^{\circledast}=M_{T}=1$.

\subsection{Bases and dimension of algebras}

We say that a subset $B \subseteq L$ is a $U$-independent set in $L$ if $B \subseteq U \neq \emptyset$ is independent in $L$.

Proposition 2.18. 1. For every independent set $Y \subseteq L$ there exists a maximal independent set $X$ in $L$ such that $Y \subseteq X$.

2. If $U \neq \emptyset$ in $L$ then for every independent set $V \subseteq U$ there exists a maximal $U$-independent set $B$ in $L$ such that $V \subseteq B$.

Proof. (i) Take an independent set $Y \subseteq L$, and let $\operatorname{In}(Y)$ denote the family of all independent sets in $L$ containing $Y$. Let $\gamma \subseteq \operatorname{In}(Y)$ be a chain. Then $Y \subseteq \bigcup \gamma$, so it is enough to show that $\bigcup \gamma$ is independent in $L$. Thus let $Z \subseteq \bigcup \gamma$ be a nonempty finite subset. Then there is $C \in \gamma$ such that $Z \subseteq C$ and hence $Z$ is independent. Due to Proposition $2.2 \cup \gamma$ is independent in $L$ and the Zorn's Lemma yields the claim.

(ii) Take $V \subseteq U$ and let $\operatorname{In}(V)$ be the family of all $U$-independent sets in $L$ containing $V$. Let $\gamma \subseteq \operatorname{In}(V)$ be a chain. Then $V \subseteq \bigcup \gamma$ and $\bigcup \gamma$ is independent in $L$. Notice now, that every $x \in \bigcup \gamma$ is a uniform element, which yields that $\bigcup \gamma$ is $U$-independent in $L$.

The above proposition suggests to take a maximal $U$-independent set of the minimal cardinality as a basis in the given lattice, and to define the dimension of this lattice as the cardinality of this basis. However, this doesn't carry the information on the decomposition into one-dimensional factors. So, we use the more adequate definition based on the notion of $\circledast$-sets (Definition 2.20).

Definition 2.19. 1. A subset $B \subseteq U \neq \emptyset$ is a $U$-basic set in $L$ if $B$ is a maximal $U$-independent set in $L$ and there is a $\circledast$-set $\left\{M_{b}^{B}\right\}_{b \in B}$.

2. A subset $B \subseteq L$ is a basic set in $L$ if $B$ is a $U$-basic set in $L$ or $B=B^{U} \cup\left\{M_{T}^{\circledast}\right\}$ is a maximal independent set in $L$ such that $B^{U} \subseteq U$ is $U$-independent and there is a $\circledast$-set $\left\{M_{b}^{B}\right\}_{b \in B^{U}} \cup\left\{M_{T}^{\circledast \circledast}\right\}$.

3. A subset $B \subseteq L$ is a basis in $L$ if $B$ is a basic set in $L$ of the minimal cardinality. 
Let us define the dimension of algebraic lattices and algebras as follows:

Definition 2.20. Let $L$ be an algebraic lattice.

1. If $0=1$ in $L$ then $\operatorname{Dim}(L)=0$.

2. $\operatorname{Dim}(L)=|B|$ if there exists a basis $B$, which is $U$-basic in $L$.

3. $\operatorname{Dim}(L)=\left|B^{U}\right|^{+}$if $B=B^{U} \cup\left\{M_{T}^{\circledast}\right\}$ is a basis in $L$ and there is no $U$-basic set in $L$.

4. If $L=\operatorname{Con} \mathbf{A}$ then we define $\operatorname{Dim}(\mathbf{A})=\operatorname{Dim}(\operatorname{Con} \mathbf{A})$.

If $L$ is locally uniform then $M_{T}=1, M_{T}^{\circledast}=0$ and then every $U$-basic set is also a basic set in $L$. If $L$ is anti-uniform then $M_{T}=0$, and then $M_{T}^{\circledast}=1$ is a one-element basic set in $L$, and then $\operatorname{Dim}(L)=0^{+}$.

ExAmPle 2.21. 1. Let $L$ be the Lattice 2 on Fig. 1 ., where $x, y, z$ are atoms. Then the set $\{x, y\}$ is a maximal $U$-independent set in $L$, but is not basic. However the sets $\{x, z\}$ and $\{y, z\}$ are $U$-basic in $L$ and they are bases of $L$, as well. Hence $\operatorname{Dim}(L)=2$.

2. Let $L$ be as Lattice 2 on Fig. 1 ., where $x, z$ are atoms and $y$ is antiuniform. Then the set $\{x, z\}$ is $U$-basic in $L$ and it is a basis of $L$ as well. Hence $\operatorname{Dim}(L)=2$.

3. Let $L$ be as Lattice 2 on Fig. 1 ., where $x, y$ are atoms and $z$ is antiuniform. Then the sets $\{x, z\}$ and $\{y, z\}$ are not $U$-basic in $L$ but they are bases of $L$. Then $\operatorname{Dim}(L)=1^{+}$.

Proposition 2.22. 1. An algebra $\mathbf{A}$ is finitely subdirectly irreducible iff $\operatorname{Dim}(\mathbf{A})=1$.

2. If $\operatorname{Dim}(\mathbf{A})=\eta$, then there exists $a \circledast$-decomposition of $\mathbf{A}$ into $\eta$ one-dimensional algebras.

3. If $\operatorname{Dim}(\mathbf{A})=\eta^{+}$, then there exists $a \circledast$-decomposition of $\mathbf{A}$ into $\eta$ one-dimensional algebras and the one algebra, which is related to the 'anti-uniform part' of $\mathbf{A}$.

\subsection{Comparison of $\circledast$-products with other product constructions - examples}

At the end of this section we present examples which show how $\circledast$-products behave in comparison with direct products, direct sums and weak direct products. The definition of a weak direct product was given by Grätzer in [5] and was studied by Walendziak [16], [17]. The weak direct product 
generalizes some kinds of direct sums and does not necessarily exist. For example, a weak direct product of an infinite set of Boolean algebras doesn't exist.

ExAmple 2.23. If $\mathbf{V}$ is the algebra induced on the vector space $\mathbf{V}$, then the only one-dimensional algebras are one-dimensional vector spaces, which are isomorphic to $\mathbf{K}^{1}$. The lattice $S u b \mathbf{V}$ of subspaces of $\mathbf{V}$ is isomorphic to the lattice of congruences $\operatorname{Con} \mathbf{V}$. The maximal subspaces omitting the given one-dimensional subspace $\mathbf{V}_{1}$ is a subspace $\mathbf{V}^{\perp}{ }_{1}$ (it corresponds uniquely to a congruence) such that $\mathbf{V} \simeq \mathbf{V}_{1} \oplus \mathbf{V}^{\perp}$. Hence, $\mathbf{V} \simeq \bigoplus\left(\mathbf{K}^{1}\right)_{i \in I} \simeq$ $\circledast\left(\mathbf{K}^{1}\right)_{i \in I}$, where $|I|$ is a linear dimension of $\mathbf{V}$.

EXAMPLE 2.24. As in vector spaces, the lattice of subgroups is isomorphic to the lattice of congruences for any Abelian group. Let $\mathbf{G}$ be a finitely generated Abelian group. Here, the only uniform subgroups(and one-dimensional groups, as well) are $\mathbf{Z}$ and $\mathbf{Z}_{p^{\alpha}}$, where $p$ is a prime number. As in the above example, any maximal subgroup in $\mathbf{G}$ omitting given uniform subgroup $\mathbf{G}_{1}$ is a subgroup $\mathbf{G}^{\perp}{ }_{1}$ such that $\mathbf{G} \simeq \mathbf{G}_{1} \oplus \mathbf{G}^{\perp}$. Hence, $\mathbf{G} \simeq \bigoplus\left(\mathbf{G}_{i}\right)_{i \in I} \simeq \circledast\left(\mathbf{G}_{i}\right)_{i \in I}$, where $\left(\mathbf{G}_{i}\right)_{i \in I}$ are one-dimensional factors in the direct decomposition of $\mathbf{G}$.

ExAmple 2.25. The only one-dimensional finite Boolean algebra is the two-element algebra 2 . Then any finite Boolean algebra $\mathbf{B} \simeq \prod(\mathbf{2})_{i \in I} \simeq$ $\circledast(\mathbf{2})_{i \in I}$, where $|B|=2^{|I|}$.

EXAmPle 2.26. Consider monounary algebras with the operation $f$ such that $f(x)=x$. For any algebra $\mathbf{A}$ the congruence lattice is isomorphic to the equivalence lattice on $A$. Hence the only one-dimensional algebras are two-element algebras, all of them are isomorphic to the two-element algebra, denoted by $\mathbf{2}$. Then

1. every three-element algebra $\mathbf{A}$ is isomorphic to a $\circledast$-product of two copies of $\mathbf{2}$ and every three-element subalgebra of $\mathbf{2} \times \mathbf{2}$ is isomorphic to $\mathbf{A}$,

2. the two-element subalgebras $\{(\mathbf{0}, \mathbf{0}),(\mathbf{1}, \mathbf{1})\},\{(\mathbf{0}, \mathbf{1}),(\mathbf{1}, \mathbf{0})\}$ are subdirect products of two copies of $\mathbf{2}$, but they are not $\circledast$-products.

Corollary 2.27. 1. If an algebra $\mathbf{A}$ is a finite product (a weak product, a direct sum) of some algebras then it is a $\circledast$-product of the same algebras. 
2. There are subdirect products which are not $\circledast$-products.

3. There are $\circledast$-products of finitely many one-dimensional algebras which are not isomorphic to the direct product of these algebras nor any weak product of these algebras.

4. $A \circledast$-product of one-dimensional algebras can be non-uniquely embedded into the direct product of these algebras.

5. Even in classes where direct sums exist, there can be $\circledast$-products of one-dimensional algebras which are not isomorphic to the direct sums of these algebras.

\section{Balanced algebraic lattices}

In this section we study properties of balanced lattices in the context of independent sets, $\circledast$-products and dimension. The notion of a balanced lattice was explored in papers of Zolotarev [18], [19] and Krempa [11], [12].

A lattice $L$ is balanced iff for any $x, y, z \in L$ the following balance condition is satisfied: if $x \wedge y=0$ and $(x \vee y) \wedge z=0$ then $(x \vee z) \wedge y=0$.

A lattice $L$ is modular iff for any $x, y, z \in L$ the following condition is satisfied: if $x \leq y$ then $x \vee(y \wedge z)=(x \vee y) \wedge z$.

Throughout this section, we assume that $L$ is an algebraic balanced lattice. Uniform lattices, modular lattices, distributive lattices and $N_{5}$ are balanced. Notice, that the balance condition refers to the bottom of the lattice, so it is enough to be 0-modular or 0-distributive to be balanced. A minimal non-balanced lattice (see [11]) is Lattice 2 on Fig. 1.

\subsection{Independent sets in balanced algebraic lattices}

First, let us consider maximal independent sets.

Proposition 3.1. 1. If $X$ is an independent set in $L$ and $y \wedge \bigvee X=0$ for some $y \in L \backslash\{0\}$, then $\{y\} \cup X$ is independent in $L$.

2. The following conditions are equivalent:

(a) $X$ is a maximal independent set in $L$,

(b) $X$ is an independent set and $\bigvee X$ is essential in $L$.

Proof. Let $X$ be independent in $L,|X|>1$ and $y \wedge \bigvee X=0$. Then for every $x \in X, y \wedge(x \vee \bigvee(X \backslash\{x\}))=0$ and $x \wedge \bigvee(X \backslash\{x\})=0$. Thus the 
balance condition yields $x \wedge(y \vee \bigvee(X \backslash\{x\}))=0$. Therefore $\{y\} \cup X$ is independent in $L$. (ii) follows directly from (i).

Notice that the balance condition is necessary in the last proposition. In Lattice 2 on Fig. 1, the set $\{x, y\}$ is maximal independent, but $x \vee y$ is not essential.

If $X$ is an independent set such that $|X|>1$ then $M_{x}^{X}$ denotes a maximal element omitting $x$ such that $\bigvee(X \backslash\{x\}) \leq M_{x}^{X}$.

Theorem 3.2. If $X$ is a maximal independent set in $L$ such that $|X|>1$ then $\left\{M_{x}^{X}\right\}_{x \in X}$ is a $\circledast$-set.

Proof. We have to show that:

1. $\bigwedge\left\{M_{x}^{X}\right\}_{x \in X}=0$,

2. $\left\{\bar{M}_{X}^{X}\right\}_{x \in X}$ is independent and $\bigvee\left\{\bar{M}_{x}^{X}\right\}_{x \in X}$ is essential in $L$, where $\bar{M}_{x}^{X}=\bigwedge\left\{M_{y}^{X}\right\}_{y \in X \backslash\{x\}}$,

3. $M_{x}^{X}$ is a maximal element omitting $\bar{M}_{x}^{X}$ and $\bigvee\left\{\bar{M}_{y}^{X}\right\}_{y \in X \backslash\{x\}}$ is essential in $M_{x}^{X}$, for every $x \in X$.

(i) Let $X$ be a maximal independent set in $L$ and $\alpha=\bigwedge\left\{M_{x}^{X}\right\}_{x \in X}$. Our claim is that $\alpha \wedge \bigvee X=0$. If it holds, then $\bigvee X$ being essential yields $\alpha=0$. By definition of $M_{x}^{X}, \alpha \wedge x=0$ for every $x \in X$. Hence there exists a maximal subset $C \subseteq X$ such that $\alpha \wedge \bigvee C=0$. If $C \neq X$ then for some $x \in X \backslash C, \bigvee C \leq \bigvee(X \backslash\{x\})$ and $(\alpha \vee \bigvee C) \wedge x \leq(\alpha \vee \bigvee(X \backslash\{x\})) \wedge x \leq$ $M_{x}^{X} \wedge x=0$. By the balance condition we get $\alpha \wedge(x \vee \vee C)=0$. It contradicts the maximality of $C$. Thus $C=X$, hence $\wedge\left\{M_{x}^{X}\right\}_{x \in X}=0$.

(ii) $\bigvee\left\{\bar{M}_{y}^{X}\right\}_{y \in X \backslash\{x\}} \wedge \bar{M}_{x}^{X} \leq M_{x}^{X} \wedge \wedge\left\{M_{y}^{X}\right\}_{y \in X \backslash\{x\}}=\wedge\left\{M_{x}^{X}\right\}_{x \in X}=0$ for every $x \in X$. Moreover, $x \leq \bar{M}_{x}^{X}$ for every $x \in X$, hence $\bigvee X \leq$ $\bigvee\left\{\bar{M}_{x}^{X}\right\}_{x \in X}$. Due to Proposition $3.1 \bigvee X$ is essential, so $\bigvee\left\{\bar{M}_{x}^{X}\right\}_{x \in X}$ is essential, too.

(iii) We get immediately that $\bar{M}_{x}^{X} \wedge M_{x}^{X}=\wedge\left\{M_{y}^{X}\right\}_{y \in X \backslash\{x\}} \wedge M_{x}^{X}=0$. Hence $M_{x}^{X}$ omits $\bar{M}_{x}^{X}$ and $x \leq \bar{M}_{x}^{X}$. By Proposition 2.3, $M_{x}^{X}$ is a maximal element omitting $\bar{M}_{x}^{X}$. To prove that $\bigvee\left\{\bar{M}_{y}^{X}\right\}_{y \in X \backslash\{x\}}$ is essential in $M_{x}^{X}$ we will show that $\bigvee(X \backslash\{x\}) \leq \bigvee\left\{\bar{M}_{y}^{X}\right\}_{y \in X \backslash\{x\}}$ is essential in $M_{x}^{X}$ for every $x \in X$. So, take any $\alpha \leq M_{x}^{X}$. Then $\alpha \vee \bigvee(X \backslash\{x\}) \leq M_{x}^{X}$. If $\alpha \wedge \bigvee(X \backslash\{x\})=0$ then by the balance condition $\bigvee X \wedge \alpha=0$ and $\bigvee X$ being essential in $L$ yields $\alpha=0$. 
Corollary 3.3. $\mathbf{A} \simeq \circledast\left\{\mathbf{A} / M_{x}^{X}\right\}_{x \in X}$ for any maximal independent set $X$ in $\operatorname{Con} \mathbf{A}$.

Proposition 3.4. 1. For any $x, M_{x} \in L, x \vee M_{x}$ is essential in $L$.

2. Let $\emptyset \neq X \subseteq L$. Then for any $M_{X}$ and $M_{X}^{\circledast}, M_{X} \vee M_{X}^{\circledast}$ is essential in L. Moreover, $M_{X}^{\circledast \circledast}=M_{X}$.

Proof. (i) If $z \wedge\left(x \vee M_{x}\right)=0$ then, by the balance condition $x \wedge\left(z \vee M_{x}\right)=$ 0 . Hence $z \vee M_{x}$ omits $x$, so $z=0$ by maximality of $M_{x}$.

(ii) Taking $x=M_{X}$ in (i) we get that $M_{X} \vee M_{X}^{\circledast}$ is essential in $L$. As $M_{X} \leq M_{X}^{\circledast \circledast}$ by definition, assume that $M_{X}<M_{X}^{\circledast \circledast}$. Then by maximality of $M_{X}$ there is an $x \in X$ such that $a=M_{X}^{\circledast \circledast} \wedge x \neq 0$. Then $a \vee M_{X} \leq M_{X}^{\circledast \circledast}$, so $\left(a \vee M_{X}\right) \wedge M_{X}^{\circledast}=0$. Thus $a \wedge M_{X} \leq x \wedge M_{X}=0$ and the balance condition yields $\left(a \vee M_{X}^{\circledast}\right) \wedge M_{X}=0$. Thus $a \vee M_{X}^{\circledast}$ omits $M_{X}$ and the maximality of $M_{X}^{\circledast}$ yields $a \vee M_{X}^{\circledast}=M_{X}^{\circledast}$. Hence we get $0 \neq a \leq M_{X}^{\circledast} \wedge M_{X}^{\circledast \circledast}$, which is impossible. Hence $M_{X}^{\circledast \circledast}=M_{X}$.

\subsection{Uniform elements in balanced algebraic lattices}

Proposition 3.5. Let $V=\left\{v_{i}\right\}_{i \in I}$ and $W=\left\{w_{i}\right\}_{i \in I}$, where $|I|>0$ be sets of uniform elements such that $v_{i} \leq w_{i}$ for every $i \in I$. Then

1. $V$ is independent in $L$ iff $W$ is independent in $L$,

2. $V$ is maximal $U$-independent in $L$ iff $W$ is maximal $U$-independent in $L$.

Proof. (i) It is obvious when $|I|=1$, so let $|I|>1$. Let $J \subseteq I$ be a finite nonempty subset of $I$ and $V^{\prime}=\left\{v_{i}\right\}_{i \in J}$ and $W^{\prime}=\left\{w_{i}\right\}_{i \in J}$. Due to Proposition 2.2 it is enough to show that $V^{\prime}$ is independent in $L$ iff $W^{\prime}$ is independent in $L$. This statement is obvious for $|J|=1$. Assume that $|J|>1$. If $W^{\prime}$ is independent then $V^{\prime}$ is independent since $v_{i} \wedge \bigvee\left(V^{\prime} \backslash\right.$ $\left.\left\{v_{i}\right\}\right) \leq w_{i} \wedge \bigvee\left(W^{\prime} \backslash\left\{w_{i}\right\}\right)=0$, for every $i \in J$. If $V^{\prime}$ is independent in $L$ then for every $i \in J, v_{i} \wedge \bigvee\left(V^{\prime} \backslash\left\{v_{i}\right\}\right)=0$. Moreover, $\bigvee\left(V^{\prime} \backslash\left\{v_{i}\right\}\right) \leq M_{i}^{V^{\prime}}$. Hence by Proposition $2.12 M_{i}^{V^{\prime}}$ is a maximal element omitting $w_{i}$, and thus $w_{i} \wedge \bigvee\left(V^{\prime} \backslash\left\{v_{i}\right\}\right)=0$. By Proposition $3.1\left\{w_{i}\right\} \cup\left(V^{\prime} \backslash\left\{v_{i}\right\}\right)$ is independent. By induction $W^{\prime}$ is independent.

(ii) If $V$ is not maximal $U$-independent in $L$ then there is $u \in U$ such that $V \cup\{u\}$ is independent. Then by (i) $W \cup\{u\}$ is independent in $L$ and 
thus $W$ is not maximal $U$-independent. Analogously, the opposite side is proved.

Proposition 3.6. For any $u \in U \neq \emptyset$, every $M_{u}^{\circledast}$ is a maximal uniform element in $L$.

Proof. Let $x \leq M_{u}^{\circledast}$. Then $(x \vee u) \wedge M_{u} \leq M_{u}^{\circledast} \wedge M_{u}=0$. If $x \wedge u=0$ then by the balance condition $\left(x \vee M_{u}\right) \wedge u=0$. So, $x \vee M_{u}$ omits $u$ and by maximality of $M_{u}, \quad x \leq M_{u}$ and $x=x \wedge M_{u} \leq M_{u}^{\circledast} \wedge M_{u}=0$. Thus $x \wedge u \neq 0$ for any $x \neq 0$. Now, let $0 \neq x, y \leq M_{u}^{\circledast}$. Then $x \wedge u \neq 0 \neq y$. As $u$ is uniform, we get that $0 \neq(x \wedge u) \wedge(y \wedge u)=(x \wedge y) \wedge u$. Hence $x \wedge y \neq 0$, so $M_{u}^{\circledast}$ is uniform. If $M_{u}^{\circledast} \leq v$ for some $v \in U$, then $M_{u}$ omits $v$, so $v=M_{u}^{\circledast}$.

The balance condition in the above proposition is necessary. As example, look at the nonbalanced Lattice 2 on Fig. 1 . and take $u=x$ and $M_{u}=z$. Then $M_{u}^{\circledast}=x \vee y$ is not uniform.

Proposition 3.7. For any $M_{U} \neq 1$, the interval $\left[M_{U}, 1\right]$ is locally uniform.

Proof. For any $x \in\left(M_{U}, 1\right]$ there is a $u \in U$ with $u \leq x$, so $M_{U} \vee u \leq x$. We will show that $M_{U} \vee u$ is uniform in the interval $\left[M_{U}, M_{U} \vee u\right]$.

If $b \in\left(M_{U}, M_{U} \vee u\right]$ then $b \wedge M_{U}^{\circledast} \neq 0$, by maximality of $M_{U}$. If $M_{U} \wedge\left(u \vee\left(b \wedge M_{U}^{\circledast}\right)\right) \neq 0$, then using the balance condition we get that $\left(M_{U} \wedge\left(b \wedge M_{U}^{\circledast}\right)\right) \vee\left(\left(M_{U} \vee\left(b \wedge M_{U}^{\circledast}\right)\right) \wedge u\right) \neq 0$. But $M_{U} \wedge\left(b \wedge M_{U}^{\circledast}\right)=0$, so $0 \neq\left(M_{U} \vee\left(b \wedge M_{U}^{\circledast}\right)\right) \wedge u \leq b \wedge u$. If $M_{U} \wedge\left(u \vee\left(b \wedge M_{U}^{\circledast}\right)\right)=0$ and if $u \wedge\left(b \wedge M_{U}^{\circledast}\right)=0$, then using the balance condition again, we get that $0=\left(M_{U} \vee u\right) \wedge\left(b \wedge M_{U}^{\circledast}\right)=b \wedge M_{U}^{\circledast}$. It contradicts the fact that $b \wedge M_{U}^{\circledast} \neq 0$, so $0 \neq u \wedge\left(b \wedge M_{U}^{\circledast}\right) \leq b \wedge u$. Thus $b \wedge u \neq 0$. Let $c \in\left(M_{U}, M_{U} \vee u\right]$. Then $0 \neq(b \wedge u) \wedge(c \wedge u)=b \wedge c \wedge u$ and $M_{U}<(b \wedge c \wedge u) \vee M_{U} \leq(b \wedge c) \vee M_{U}=b \wedge c$. Hence $b \wedge c>M_{U}$ and hence $M_{U} \vee u$ is uniform in the interval $\left[M_{U}, M_{U} \vee u\right.$ ].

Corollary 3.8. Let $\mathbf{A}$ be a congruence balanced algebra with $0 \neq M_{U} \neq 1$ in Con $\mathbf{A}$. Then $\mathbf{A} \simeq \mathbf{A} / M_{U} \circledast \mathbf{A} / M_{U}^{\circledast}$, where $\mathbf{A} / M_{U}$ is congruence locally uniform.

The previous result is important because it allows to distinguish a locally uniform part of the given algebra. Unfortunately, this locally uniform part depends on the choice of $M_{U}$. Notice also that $\left[M_{U}, 1\right]$ need not to be a balanced lattice. 


\subsection{Bases in balanced algebraic lattices}

A Goldie basis in $L$ is a maximal $U$-independent set in $L$. According to Krempa [11] every balanced locally uniform lattice has a Goldie basis. It is also known that Goldie bases in the given balanced locally uniform lattice have the same cardinality (see [18]). Earlier analogous results for modular lattices were given in [9]. The Goldie dimension of a lattice $L$ was defined as the cardinality of any Goldie basis in $L$. More on the Goldie dimension can be found in [19], [12], [8].

Proposition 3.9. Assume that $L$ is a balanced locally uniform algebraic lattice. Then

1. $B$ is a $U$-basic set in $L$ iff $B$ is a maximal independent set in $L$,

2. $B$ is a basis in $L$ iff $B$ is a Goldie basis in $L$,

3. $\operatorname{Dim}(L)$ is equal to the Goldie dimension of $L$.

Proof. (i) is an immediate consequence of Proposition 3.1.

(ii) Let $B$ be a Goldie basis in $L$, so it is a maximal $U$-independent set in $L$. If $0 \neq x \in L$ and $x \wedge \bigvee B=0$, then $L$ being locally uniform yields $u \leq x$ for some $u \in U$ and $u \wedge \bigvee B=0$. Then $\{u\} \cup B$ is a $U$-independent set, which contradicts the maximality of $B$. Hence $\bigvee B$ is essential in $L$. Thus $B$ is a $U$-basic set in $L$. As all Goldie bases have equal cardinality we conclude that $B$ is a basis in $L$ and thus (iii) is true.

This yields that every congruence locally uniform balanced algebra A is a $\circledast$-product of $\operatorname{Dim}(\mathbf{A})$ one-dimensional algebras. At the end of this section we give the following basis existence theorem for any balanced algebraic lattice.

THEOREM 3.10. Every nontrivial balanced algebraic lattice has a basis.

Proof. Let $L$ be a balanced algebraic lattice. If $L$ is locally uniform, then $L$ has a basis. If $L$ is anti-uniform, then $\{1\}$ is a basis. If $L$ is not locally uniform nor anti-uniform then by definition, the interval $\left[0, M_{T}\right]$ is locally uniform for any $M_{T}$. Thus $\left[0, M_{T}\right]$ has a basis $B^{T}$. If $B^{T}$ is a $U$-basic set in $L$ then it is a basic set in $L$, if not then $B^{T}$ can be extended to a maximal $U$-independent set $C=B^{T} \cup B^{\prime}$, due to Proposition 2.18. If $0 \neq \alpha=\bigvee B^{\prime} \wedge M_{T}$ then $\bigvee B^{T}$ being essential in $\left[0, M_{T}\right]$ yields $0 \neq$ $\alpha \wedge \bigvee B^{T} \leq \bigvee B^{\prime} \wedge \bigvee B^{T}=0$. A contradiction. Thus $\bigvee B^{\prime} \wedge M_{T}=0$ and hence there exists an $M_{T}^{\circledast} \geq \bigvee B^{\prime}$. 
We are going to show that $B=\left\{M_{T}^{\circledast}\right\} \cup B^{T}$ is a maximal independent set in $L$. As $M_{T}^{\circledast} \wedge \bigvee B^{T} \leq M_{T}^{\circledast} \wedge M_{T}=0$ we use Proposition 3.1 to get that $B$ is independent in $L$. We will show that $\bigvee B^{T} \vee M_{T}^{\circledast}$ is essential in $L$. Due to Proposition 3.4 it is enough to show that $M_{T}^{\circledast}$ is a maximal element omitting $\bigvee B^{T}$, so assume that $M_{\bigvee B^{T}}>M_{T}^{\circledast}$. Then $M_{\bigvee B^{T}} \wedge M_{T}>0$. But $\bigvee B^{T}$ is essential in $\left[0, M_{T}\right]$, hence $0 \neq M_{\bigvee B^{T}} \wedge M_{T} \wedge \bigvee B^{T}=M_{\bigvee B^{T}} \wedge$ $\bigvee B^{T}=0$. A contradiction. Finally, by Theorem $3.2\left\{M_{b}\right\}_{b \in B}$ is a $\circledast$-set and by Proposition 3.4 $M_{T}=M_{T}^{\circledast \circledast}$. Thus $B$ is a basic set. Finally, there exists a basis in $L$, which is any basic set of minimal cardinality.

\section{Modular algebraic lattices}

In this section let $L$ represent a modular algebraic lattice with nonempty sets $U$ and $T$. Every modular lattice is balanced, so all the properties described in the previous section hold in modular lattices. Goldie dimension in modular lattices was investigated in [8], [9], [14].

Recall some properties of modular lattices.

M1 A lattice $L$ is modular iff it satisfies the identity: $(x \vee y) \wedge(x \vee z)=x \vee(y \wedge(x \vee z))$

M2 For any modular lattices $L$ the Isomorphism Theorem holds, i.e. for any $a, b \in L$ the intervals $[a, a \vee b]$ and $[a \wedge b, b]$ are isomorphic and the isomorphism is $\varphi_{b}(x)=x \wedge b$, while the inverse isomorphism is $\psi_{a}(x)=x \vee a$.

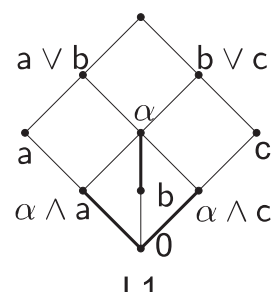

L1

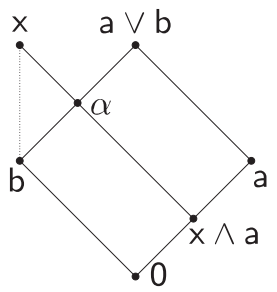

L2

Fig. 2 . 
The modularity of a lattice and especially, the Isomorphism Theorem, allow to simplify results of previous sections and to obtain some more interesting properties concerning dimension and $\circledast$-decompositions. We give below two lemmas useful in our proofs.

Lemma 4.1. Let $a, b, c \in L \backslash\{0\}$ be such that $a \wedge b=a \wedge c=b \wedge c=0$ and let $\alpha=(a \vee b) \wedge(c \vee b)>b$. Then $\alpha \wedge a \neq 0$ and $\alpha \wedge c \neq 0$ and the intervals $[0, \alpha \wedge a],[b, \alpha],[0, \alpha \wedge c]$ are isomorphic (see L1 Fig. 2).

Proof. Let $a, b, c \in L$ be such that $a \wedge b=a \wedge c=a \wedge c=0$ and $\alpha>b$. Then by property M1 $\alpha=(a \vee b) \wedge(c \vee b)=b \vee(a \wedge(c \vee b))=b \vee(c \wedge(a \vee b))$ and $(\alpha \wedge a) \vee b=((a \vee b) \wedge(c \vee b) \wedge a) \vee b=((c \vee b) \wedge a) \vee b=\alpha$. By assumption we have that $(\alpha \wedge a) \wedge b=\alpha \wedge(a \wedge b)=0$. Hence, by M2 $[0, \alpha \wedge a] \simeq[b, \alpha]$. Analogously, $[0, \alpha \wedge c] \simeq[b, \alpha]$. Thus $\alpha \wedge a \neq 0 \neq \alpha \wedge c$.

Lemma 4.2. Let $a, b, x \in L \backslash\{0\}$ be such that $a \wedge b=0, x \wedge a>0$ and $x>b$ and let $\alpha=(x \wedge a) \vee b$ (see L2 Fig. 2). Then $b<\alpha=x \wedge(a \vee b) \leq a \vee b$.

Proof. If $a \wedge b=0$ and $x \wedge a>0$ then $b<(x \wedge a) \vee b$. Modularity of $L$ and $x>b$ imply $(x \wedge a) \vee b=x \wedge(a \vee b) \leq a \vee b$.

The next proposition shows that it is possible to distribute uniform elements (at least a part of each of them) to $M_{U}^{\circledast}=M_{T}$ and anti-uniform elements (at least a part of each of them) to $M_{U}=M_{T}^{\circledast}$, independently of the choice of $M_{U}$ and $M_{U}^{\circledast}$.

Proposition 4.3. For any $M_{U}$ and $M_{U}^{\circledast}$ the following holds:

1. $M_{U} \wedge t \neq 0$ for every $t \in T$,

2. $M_{U}^{\circledast} \wedge u \neq 0$ for every $u \in U$,

3. $M_{U}^{\circledast} \wedge t=0$ for every $t \in T$,

4. there exist $M_{T}$ and $M_{T}^{\circledast}$ such that $M_{U}^{\circledast}=M_{T}$ and $M_{U}=M_{T}^{\circledast}$, and conversely.

Proof. (i) If $M_{U} \wedge t=0$ then $M_{U} \vee t>M_{U}$. By maximality of $M_{U}$ there exists an $u \in U$ such that $u \leq M_{U} \vee t$. Use Lemma 4.1 where $a=u, b=M_{U}, c=t$. Then $\alpha=\left(u \vee M_{U}\right) \wedge\left(t \vee M_{U}\right)=M_{U} \vee\left(u \wedge\left(t \vee M_{U}\right)\right)=$ $u \vee M_{U}>M_{U}$. Hence $\alpha \wedge u=\left(M_{U} \vee u\right) \wedge u=u$ and $\alpha \wedge t=t^{\prime} \leq t$ and $[0, u] \simeq\left[0, t^{\prime}\right]$ what is impossible as $u$ is uniform while $t^{\prime}$ is anti-uniform.

(ii) Assume that $M_{U}^{\circledast} \wedge u=0$ for some $u \in U$. Then $M_{U}^{\circledast} \vee u>M_{U}^{\circledast}$ and hence $M_{U} \wedge\left(M_{U}^{\circledast} \vee u\right)>0$. Moreover, $u \wedge\left(M_{U} \wedge\left(M_{U}^{\circledast} \vee u\right)\right) \leq u \wedge M_{U}=0$, so 
$u \vee\left(M_{U} \wedge\left(u \vee M_{U}^{\circledast}\right)\right)>u$. Using Lemma 4.1 where $a=M_{U}, b=u, c=M_{U}^{\circledast}$ we get that $\alpha=\left(u \vee M_{U}\right) \wedge\left(u \vee M_{U}^{\circledast}\right)=u \vee\left(M_{U} \wedge\left(u \vee M_{U}^{\circledast}\right)\right)>u$. Hence $\left[0, \alpha \wedge M_{U}\right] \simeq\left[0, \alpha \wedge M_{U}^{\circledast}\right]$. As $\alpha \wedge M_{U} \leq M_{U}$ is anti-uniform, $\alpha \wedge M_{U}^{\circledast}$ is also anti-uniform. Then (i) implies $0<M_{U} \wedge\left(\alpha \wedge M_{U}^{\circledast}\right)=M_{U} \wedge \alpha \wedge M_{U}^{\circledast}=0$. (iii) If $M_{U}^{\circledast} \wedge t \neq 0$ for some $t \in T$ then $t^{\prime}=M_{U}^{\circledast} \wedge t \leq M_{U}^{\circledast}$ is anti-uniform. Thus by (i) $0 \neq M_{U} \wedge t^{\prime} \leq M_{U} \wedge M_{U}^{\circledast}=0$, which is impossible.

(iv) $M_{U}^{\circledast}$ omits every anti-uniform, so $M_{U}^{\circledast} \leq M_{T}$ for some $M_{T}$, but every $M_{T}$ omits $M_{U}$, so by maximality of $M_{U}^{\circledast}, M_{U}^{\circledast}=M_{T}$. Finally, by (i) $M_{T}^{\circledast}=M_{U}^{\circledast \circledast}=M_{U}$.

We will show that in modular lattices all $\left[M_{U}, 1\right]$ have the same dimension independently of the choice of $M_{U}$. First, notice that for any $M_{U}, M_{T}$, $\left[0, M_{T}\right] \simeq\left[M_{U}, M_{U} \vee M_{T}\right] \subseteq\left[M_{U}, 1\right]$ and $\left[0, M_{U}\right] \simeq\left[M_{T}, M_{U} \vee M_{T}\right] \subseteq$ $\left[M_{T}, 1\right]$.

Proposition 4.4. For any $M_{U}, M_{T}$ :

1. the intervals $\left[M_{U}, 1\right]$ and $\left[0, M_{T}\right]$ are locally uniform,

2. the intervals $\left[M_{T}, 1\right]$ and $\left[0, M_{U}\right]$ are anti-uniform.

Proof. (i) By Proposition $3.7\left[M_{U}, 1\right]$ is locally uniform, so $\left[M_{U}, M_{U} \vee\right.$ $\left.M_{T}\right]$ is locally uniform, too.

(ii) Obviously, $\left[0, M_{U}\right]$ is anti-uniform. Assume that $x$ is uniform in $\left[0, M_{T}\right]$ then $x \wedge M_{U}>0$ and we can use Lemma 4.2, where $a=M_{U}, b=M_{T}, x=x$ getting $M_{T}<\left(x \wedge M_{U}\right) \vee M_{T}=x \wedge\left(M_{T} \vee M_{U}\right)$. Then $x \wedge\left(M_{T} \vee M_{U}\right) \leq x$ is uniform in $\left[M_{T}, M_{U} \vee M_{T}\right]$ and . $\left(x \wedge\left(M_{T} \vee M_{U}\right)\right) \wedge M_{U}=x \wedge M_{U}$ is uniform in $\left[0, M_{U}\right]$. A contradiction.

Lemma 4.5. Let $x, y \in L \backslash\{0\}$ and $x \wedge y=0$.

1. Let the interval $[0, x]$ be locally uniform. Then if $B$ is a basis in $[0, x]$ then $\{b \vee y\}_{b \in B}$ is a basis in $[y, x \vee y]$, and if $C$ is a basis in $[y, x \vee y]$ then $\{c \wedge x\}_{c \in C}$ is basis in $[0, x]$.

2. Let the interval $[y, 1]$ be locally uniform and $x=M_{y}, y=M_{x}$. Then if $B$ is a basis in $[y, 1]$ then $\{b \wedge(x \vee y)\}_{b \in B}$ is a basis in $[y, x \vee y]$, and if $C$ is a basis in $[y, x \vee y]$ then $C$ is a basis in $[y, 1]$.

Proof. (i) By M2 $[0, x] \simeq[y, x \vee y]$, so for any basis $B$ in $[0, x]$ the set of isomorphic images $\left\{\psi_{y}(b)\right\}_{b \in B}=\{b \vee y\}_{b \in B}$ is a basis in $[y, x \vee y]$ and conversely, if $C$ is a basis in $[y, x \vee y]$ then $\left\{\varphi_{x}(c)\right\}_{c \in C}=\{c \wedge x\}_{c \in C}$ is a basis in $[0, x]$. 
(ii) Let $B$ be a basis in $[y, 1]$ then $b>y$ for every $b \in B$. The assumption that $y=M_{x}$ yields $b \wedge x>0$ and by Lemma $4.2 y<(b \wedge x) \vee y=b \wedge(x \vee y) \leq b$ for every $b \in B$. Thus $\{b \wedge(x \vee y)\}_{b \in B}$ is a basis in $[y, 1]$. As $b \wedge(x \vee y) \leq x \vee y$

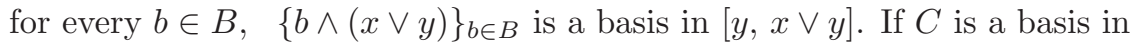
$[y, x \vee y]$ then $\bigvee C$ is essential in $[y, x \vee y]$ and $C$ is independent in $[y, 1]$. We will show that $\bigvee C$ is essential in $[y, 1]$. Take any $z>y$. Then $z \wedge x>0$ since $y=M_{x}$. Hence $y<(z \wedge x) \vee y=z \wedge(x \vee y) \leq x \vee y$ by Lemma 4.2. Finally, $\bigvee C \wedge z \geq \bigvee C \wedge(z \wedge(x \vee y))>y$.

Proposition 4.6. For any $M_{U}$ and $M_{T}$ if $B$ is a basis in $\left[M_{U}, 1\right]$ then the set $\left\{b \wedge\left(M_{U} \vee M_{T}\right)\right\}_{b \in B}$ is a basis in $\left[M_{U}, M_{U} \vee M_{T}\right]$. And then $\left\{b \wedge M_{T}\right\}_{b \in B}$ is a basis in $\left[0, M_{T}\right]$. And if $C$ is a basis in $\left[M_{U}, M_{U} \vee M_{T}\right]$ then $C$ is a basis in $\left[M_{U}, 1\right]$.

Proof. Notice that $M_{U}, M_{T}$ satisfy the assumptions of Lemma 4.5.

Corollary 4.7. For any $M_{U}$ and $M_{T}, \operatorname{Dim}\left(\left[M_{U}, 1\right]\right)=\operatorname{Dim}\left(\left[0, M_{T}\right]\right)$. Hence, for any congruence modular algebra $\mathbf{A}$ with $\emptyset \neq U \subseteq C$ Con $\mathbf{A}$, the quotients $\mathbf{A} / M_{U}$ have equal dimensions independently of the choice of $M_{U}$.

\section{1. $\circledast$-decompositions in modular algebraic lattices}

This subsection is devoted to describing the $\circledast$-decompositions of congruence modular algebras.

Proposition 4.8. For any maximal $U$-independent set $B$ in $L$ and any $M_{U}, M_{T}$ it holds that:

1. $B^{\prime}=\left\{b \wedge M_{T}\right\}_{b \in B}$ is a basis in $\left[0, M_{T}\right]$. Therefore, all the maximal $U$-independent sets in $L$ are of the same cardinality,

2. for every $b \in B$ there exists an such that $M_{b} \geq M_{U} \vee \bigvee\left(B^{\prime} \backslash\left\{b \wedge M_{T}\right\}\right)$ and $M_{U}=\bigwedge\left\{M_{b}\right\}_{b \in B}$,

3. $B^{\prime} \cup\left\{M_{U}\right\}$ is a basis in $L$.

Proof. Let $B$ be a maximal $U$-independent set in $L$.

(i) Then $B^{\prime}$ is a maximal $U$-independent set in $L$ and $0<b \wedge M_{T} \leq$ $M_{T}$. Hence $B^{\prime}$ is an independent set in $\left[0, M_{T}\right]$. By Proposition 3.1 it is enough to show that $\bigvee B^{\prime}$ is essential in $\left[0, M_{T}\right]$. So, let $0<x \leq M_{T}$ and $x \wedge \bigvee B^{\prime}=0$. Then there is a uniform element $u \leq x$ and $u \wedge \bigvee B^{\prime}=0$. Hence $\{u\} \cup B^{\prime}$ and $\{u\} \cup B$ are $U$-independent sets in $L$. It contradicts the maximality of $B$. As $\left[0, M_{T}\right]$ is locally uniform all the bases in $\left[0, M_{T}\right]$ are of the same cardinality. 
(ii) Let $B^{\prime}$ be as in (i). Then $B^{\prime}$ is independent in $L$ and $M_{U} \wedge \bigvee B^{\prime} \leq M_{U} \wedge$ $M_{T}=0$ hence $B^{\prime} \cup\left\{M_{U}\right\}$ is independent in $L$ and for every $b \in B$ we can take an $M_{b^{\prime}}$ such that $M_{U} \vee \bigvee\left(B^{\prime} \backslash\left\{b^{\prime}\right\}\right) \leq M_{b^{\prime}}$. If $M_{U}<\bigwedge\left\{M_{b^{\prime}}\right\}_{b \in B}$ then $0<\bigwedge\left\{M_{b^{\prime}}\right\}_{b \in B} \wedge M_{T}=\bigwedge\left\{M_{b^{\prime}} \wedge M_{T}\right\}_{b \in B} \leq 0$. Thus $M_{U}=\bigwedge\left\{M_{b^{\prime}}\right\}_{b \in B}$. As $b^{\prime} \leq b$, Proposition 2.12 yields $M_{b^{\prime}} \in \mathfrak{M}_{b}$ for every $b \in B$.

(iii) Let $B^{\prime}$ be as in (i). Then $\left\{M_{U}\right\} \cup B^{\prime}$ is independent in $L$ and $\bigvee B^{\prime}$ is essential in $\left[0, M_{T}\right]$. We are going to show that $M_{U} \vee \bigvee B^{\prime}$ is essential in $L$. Let $x \in L \backslash\{0\}$ and $x \wedge M_{U}=0$. Then by Proposition $4.3 x \notin T$ is not and hence there is a uniform element $u \leq x$ and $0<u \wedge M_{T} \leq x \wedge M_{T}$. Hence $x \wedge \bigvee B^{\prime}>0$, so $\left\{M_{U}\right\} \cup B^{\prime}$ is a maximal independent set in $L$. By Theorem $3.2\left\{M_{b^{\prime}}\right\}_{b \in B} \cup\left\{M_{T}\right\}$ is a $\circledast$-set in $L$, where $\left\{M_{b^{\prime}}\right\}_{b \in B}$ are as in (ii). Thus $\left\{M_{U}\right\} \cup B^{\prime}$ is a basic set and by (i) it is also a basis in $L$.

Proposition 4.9. Let $B$ be a $U$-basic set in $L$. Then $L$ is locally uniform and $B$ is a basis of $L$.

Proof. By definition of a $U$-basic set the $U \neq \emptyset$ and there exists a $\circledast$-set $\left\{M_{b}^{B}\right\}_{b \in B}$. Then $L$ is not anti-uniform. Assume that $T \neq \emptyset$. Then $B^{\prime}=\left\{b \wedge M_{T}\right\}_{b \in B}$ is a $U$-basic set in $L$ due to Proposition 2.12. Moreover, $0<b^{\prime} \leq b \leq \bar{M}_{b}^{B} \leq M_{b}^{\circledast}$. As $M_{b}^{\circledast}$ is uniform, $\bar{M}_{b}^{B}$ is uniform, too. Moreover, $\left\{\bar{M}_{b}^{B}\right\}_{b \in B}$ is a maximal independent set in $L$ and thus $B^{\prime}$ is a maximal independent set in $L$. This contradicts the (iii) of Proposition 4.8. Thus $T=\emptyset$ what means that $L$ is locally uniform and $B$ is a basis of $L$.

Proposition 4.10. $\quad$ 1. Let $r \in L \backslash\{0\}$ be a non-essential element in $L$ such that the interval $[r, 1]$ is a uniform lattice. Then every $M_{r}$ is a maximal uniform element in $L$ and $r=M_{r}^{\circledast}$.

2. Let $\left\{y_{i}\right\}_{i \in I} \subseteq L$ be $a \circledast$-set in $L$ such that $\left[y_{i}, 1\right]$ is a uniform lattice for every $i \in I$. Then $\left\{\bar{y}_{i}\right\}_{i \in I}$ is a basis in $L$.

3. For any algebra $\mathbf{A}$, if $\left\{y_{i}\right\}_{i \in I} \subseteq$ Con $\mathbf{A}$ is a $\circledast$-set such that $\left[y_{i}, 1\right]$ is a uniform lattice for every $i \in I$, then $\mathbf{A} \simeq \circledast\left(\mathbf{A}_{i}\right)_{i \in I}$ and $\operatorname{Dim}\left(\mathbf{A}_{i}\right)=$ 1 for every $i \in I$.

4. If $\mathbf{A} \simeq \circledast\left(\mathbf{A}_{i}\right)_{i \in I}$ and $\operatorname{Dim}\left(\mathbf{A}_{i}\right)=1$ for every $i \in I$, then $\left\{k e r \pi_{i}\right\}_{i \in I}$ is $a \circledast$-set in Con $\mathbf{A}$ and $\left\{\overline{k e r \pi}_{i}\right\}_{i \in I}$ is a basis in Con $\mathbf{A}$.

Proof. (i) Let $[r, 1]$ be a uniform lattice for some non-essential $r \neq 0$. Then $M_{r} \neq 0$ and $r \wedge M_{r}=0$, so $r \vee M_{r}>r$. Thus we get a uniform lattice $\left[r, r \vee M_{r}\right] \simeq\left[0, M_{r}\right]$, so $M_{r} \in U$. Assume that $u \geq M_{r}$ for some $u \in U$. 
As $r \wedge M_{r}=0$ we get $r \wedge u=0$ (by Proposition 2.12), so $u \neq M_{r}$, what means that $M_{r}$ is a maximal uniform element in $L$. By definition $r \leq M_{r}^{\circledast}$. If $r<M_{r}^{\circledast}$ then $[r, 1]$ being uniform yields $r<M_{r}^{\circledast} \wedge\left(r \vee M_{r}\right)$. Hence $0=r \wedge M_{r}<\left(M_{r}^{\circledast} \wedge\left(r \vee M_{r}\right)\right) \wedge M_{r} \leq M_{r}^{\circledast} \wedge M_{r}=0$. A contradiction.

(ii) If $|I|=1$ then $y_{i}=0$ and then $\bar{y}_{i}=1$. If $|I|>1$ then every $\bar{y}_{i}$ is a nonzero non-essential element in $L$. Then by (i) $M_{y_{i}}$ is a maximal uniform element and $y_{i}=M_{y_{i}}^{\circledast}$. This yields that $\bar{y}_{i}=\bigwedge\left\{M_{y_{j}}^{\circledast}\right\}_{j \in I \backslash\{i\}} \leq M_{y_{i}}$. Thus every $\bar{y}_{i}$ is uniform and by definition of a $\circledast$-set, $\left\{\bar{y}_{i}\right\}_{i \in I}$ is a $U$-basic set.

(iii) By assumption $\mathbf{A} \simeq \circledast\left(\mathbf{A} / y_{i}\right)_{i \in I}$. As every $\left[y_{i}, 1\right]$ is a uniform lattice, we have that every $\mathbf{A} / y_{i}$ is a one-dimensional algebra.

(iv) is an easy conclusion from (i)-(iii).

ExAmPle 4.11 . Let $R$ be a commutative ring with unity. The ideal lattice $I(R)$ of $R$ is modular. If $P \in I(R)$ is a prime ideal in $R$ then the interval $[P, 1]$ is a uniform lattice, hence the integral domain $R / P$ is a onedimensional commutative ring with unity. If $\left\{P_{i}\right\}_{i \in I} \subseteq I(R)$ is a $\circledast$-set consisting of prime ideals in $I(R)$ then $R \simeq \circledast\left(R / P_{i}\right)_{i \in I}$ of its integral domains. Moreover, $\left\{\bar{P}_{i}\right\}_{i \in I}$ is a $U$-basic set in $I(R)$. As it can be seen from Theorem $4.12\left(\bar{P}_{i}\right)_{i \in I}$ is a basis in $I(R)$.

Summarizing the results of this section we obtain the following theorem.

TheOrem 4.12. Assume that $\mathbf{A}$ is a congruence modular algebra.

1. The following conditions are equivalent:

(a) $\operatorname{Dim}(\mathbf{A})=\eta \neq 0$, where $\eta$ is a cardinal number,

(b) $\mathbf{A} \simeq \circledast\left(\mathbf{A}_{i}\right)_{i \in I}$, where $\operatorname{Dim}\left(\mathbf{A}_{i}\right)=1$ for every $i \in I$ and $|I|=\eta$,

(c) $\mathbf{A}$ is congruence locally uniform and $|B|=\eta$ for every basis $B$ in $\operatorname{Con} \mathbf{A}$.

2. The following conditions are equivalent:

(a) $\operatorname{Dim}(\mathbf{A})=\eta^{+}$, where $\eta$ is a cardinal number,

(b) Con $\mathbf{A}$ is not locally uniform and $|B|=\eta$ for every maximal $U$-independent set $B$ in $C o n \mathbf{A}$,

(c) $\mathbf{A} \simeq \circledast\left(\mathbf{A}_{b}\right)_{b \in B \cup \dot{\cup} \tau\}}$, where $B$ is any maximal $U$-independent set in $\operatorname{Con} \mathbf{A}$ and $\operatorname{Dim}\left(\mathbf{A}_{b}\right)=1$ for every $b \in B$, while $\operatorname{Dim}\left(\mathbf{A}_{\tau}\right)=$ $0^{+}$. 


\subsection{Distributive algebraic lattices}

Let as consider in this subsection algebraic distributive lattice $L$. In this case all the facts concerning modular lattices are also true. The difference is that in distributive lattice the maximal element omitting the given set is uniquely determined. As every lattice is congruence distributive the results obtained here are important in the lattice theory and logic.

Proposition 4.13. For any $X \subseteq L$ there exists exactly one $M_{X}$ and exactly one $M_{X}^{\circledast}$.

Proof. Let $M$ and $N$ be maximal elements in $L$ omitting $X$. Then we have $x \wedge M=x \wedge N=0$ and $x \wedge(M \vee N)=(x \wedge M) \vee(x \wedge N)=0$ for every $x \in X$. Thus $M \vee N$ omits $X$ and maximality of $M$ and $N$ yields $M=N$. The proof of the second part is similar.

Corollary 4.14. 1. For any $u \in U$ it holds that $M_{u}^{\circledast}$ is the only maximal uniform element such that $u \leq M_{u}^{\circledast}$.

2. $M_{U}$ is the only maximal anti-uniform element.

3. $M_{T}=M_{U}^{\circledast}$ is unique.

Proposition 4.15. If $L$ is an algebraic distributive locally uniform lattice then the set $V$ off all maximal uniform elements in $L$ is a basis in $L$.

Proof. Let $B \subseteq U$ be a basis in $L$ and let $u \in V$. We will show that there exists $b \in B$ such that $u \wedge b \neq 0$. If it holds then $u \wedge b \leq u=M_{u \wedge b}^{\circledast}=b$. If $|B|=1$ then $b \in B$ is essential, so $u \wedge b \neq 0$. If $|B|>1$ then $u \wedge \bigvee B>0$ Assume that $u \wedge b=0$ for every $b \in B$. Let $C \subset B$ be a maximal subset such that $u \wedge \bigvee\{c\}_{c \in C}=0$. Let $b \in B \backslash C$. Then $0 \neq u \wedge(b \vee \bigvee C)=$ $(u \wedge b) \vee(u \wedge \bigvee C)$. Hence $u \wedge b \neq 0$.

Theorem 4.12 can be directly applied to congruence distributive algebras taking the set of all maximal uniform elements $V$ as a maximal $U$-independent set.

TheOrem 4.16. Let $\mathbf{A}$ be a congruence distributive algebra and let $V$ be the set of all maximal uniform elements in Con $\mathbf{A}$.

1. The following conditions are equivalent:

(a) $\operatorname{Dim}(\mathbf{A})=\eta \neq 0$, where $\eta$ is a cardinal number,

(b) A is congruence locally uniform and $|V|=\eta$, 
(c) $V \neq \emptyset$ and $\mathbf{A} \simeq \circledast\left(\mathbf{A}_{v}\right)_{v \in V}$ and $\operatorname{Dim}\left(\mathbf{A}_{v}\right)=1$ for every $v \in V$. Moreover, such a decomposition is unique.

2. The following conditions are equivalent:

(a) $\operatorname{Dim}(\mathbf{A})=\eta^{+}$, where $\eta$ is a cardinal number,

(b) Con $\mathbf{A}$ is not locally uniform and $|V|=\eta$,

(c) $\mathbf{A} \simeq \circledast\left(\mathbf{A}_{v}\right)_{v \in V \cup \dot{ }\{\tau\}}$ and for every $v \in V, \operatorname{Dim}\left(\mathbf{A}_{v}\right)=1$ and $\operatorname{Dim}\left(\mathbf{A}_{\tau}\right)=0^{+}$, where $\mathbf{A}_{\tau} \simeq \mathbf{A} / M_{T}$. Moreover, such a decomposition is unique.

\section{References}

[1] G. Birkhoff, On the structure of abstract algebras, Proc. Cambridge Philos. Soc. 31 (1935), pp. 433-454.

[2] G. Birkhoff, Lattice Theory. American Mathematical Society Colloquium Publications, vol. 25, American Mathematical Society, New York, (1948).

[3] S. Burris, H. P. Sankappanavar, A Course in Universal Algebra, Springer-Verlag, 1981.

[4] A. W. Goldie, The structure of prime rings under ascending chain conditions, Proc. London Math. Soc. (3) 8(1958), pp. 589-608.

[5] G. Grätzer, General Lattice Theory. Second edition. Birkhauser Verlag, Basel (1998).

[6] G. Grätzer, E. T. Schmidt, Characterizations of congruence lattices of abstract algebras, Acta Sci. Math. (Szeged) 24(1963), pp. 34-59.

[7] P. Grzeszczuk, J. Okniński, E. R. Puczyłowski, Relations between some dimensions of modular lattices, Comm. Algebra 17(1989), pp. 1723-1737.

[8] P. Grzeszczuk, E. R. Puczyłowski, On Goldie and dual Goldie dimensions, J. Pure Appl. Algebra 31(1984), pp. 47-54.

[9] P. Grzeszczuk, E. R. Puczyłowski, On infnite Goldie dimension of modular lattices and modules, J. Pure Appl. Algebra 35(1985), pp. 151-155.

[10] T. Katrinák, S. El-Assar, Algebras with Boolean and Stonean congruence lattices, Acta Math. Hungar. 48 (1986), pp. 301-31.

[11] J. Krempa, On lattices, modules and groups with many uniform elements, Algebra Discrete Math. 1 (2004), pp. 75-86.

[12] J. Krempa, B. Terlikowska-Osłowska, On uniform dimension of lattices, Contributions to General Algebra 9, Holder-Pichler-Tempsky, Wien, 1995, pp. 219-230. 
[13] R. N. McKenzie, G. F. McNulty, W. F. Taylor, Algebras, Lattices, Varieties, Vol. 1, The Wadsworth \& Brooks/Cole Mathematics Series. Wadsworth \& Brooks/Cole Advanced Books \& Software, Monterey, California, (1987).

[14] E. R. Puczyłowski, A linear property of Goldie dimension of modules and modular lattices, J. Pure Appl. Algebra 215 (2011), pp. 1596-1605.

[15] S. Roman, Lattices and Ordered Sets, Springer-Verlag, New York, 2008.

[16] A. Walendziak, On direct decompositions of lattices, bf Algebra Universalis, 37 (1997), pp. 185-190.

[17] A. Walendziak, Decompositions in lattices and some representations of algebras, Dissertationes Mathematicae 392, (2001).

[18] A. P. Zolotarev, On balanced lattices and Goldie dimension of balanced lattices, Siberian Math. J. 35:3 (1994), pp. 539-546.

[19] A. P. Zolotarev, Direct decompositions of elements, and Goldie numbers in balanced lattices, Russian Math. (Iz. VUZ), 36:10 (1992), pp. 19-27.

Department of Mathematics and Computer Science University of Warmia and Mazury, Olsztyn, Poland bstar@uwm.edu.pl 